\title{
Threefold spin helicity as possible origin of $S U(3)$ gauge symmetry
}

\author{
E. Marsch $^{1, \mathrm{a}} \mathbb{D}$, Y. Narita ${ }^{2, b}$ \\ ${ }^{1}$ Extraterrestrial Physics Group, Institute for Experimental and Applied Physics, Christian Albrechts \\ University at Kiel, Leibnizstraße 11, D-24118 Kiel, Germany \\ 2 Space Research Institute, Austrian Academy of Sciences, Schmiedlstraße 6, A-8042 Graz, Austria
}

Received: 9 May 2021 / Accepted: 6 June 2021

(C) The Author(s) 2021

\begin{abstract}
In this paper, the notion of spin helicity is generalized into threefold spin helicity. It appears to be a useful means to extend the standard Dirac equation to describe coloured fermions. The threefold generalization of helicity is derived from the mass shell condition, spin, and kinetic helicity in a natural way. It is found that the three different types of helicities are associated with the rotation degrees of freedom in 3-D coordinate space. Moreover, threefold helicity can by unitary transformation be connected with the empirical $S U(3)$ colour symmetry of the quarks, and thus be brought into the mathematical form of the $S U(3)$ Yang-Mills gauge theory of the standard model. This offers an alternative picture of the physical origin of quark symmetry in compliance with the Coleman-Mandula theorem.
\end{abstract}

\section{Introduction}

Although the existence of three colours of quarks is empirically well established (see, for example, the experimental textbook by Machner [1]), and the related theory of quantum chromodynamics (QCD) is a celebrated building block of the theoretical standard model (SM) of elementary particle physics [2-4], the deeper physical origin of the three colour degrees remains an unexplained theoretical mystery of modern physics. A recent attempt to explain the origin of flavour and colour was made by Marsch [5] on the basis of permutation symmetries with respect to the Pauli matrices appearing in the famous Dirac gamma matrices [6]. This permutation symmetry is suggested by the fundamental $s u(2) \otimes s u(2)$ symmetry of the Lorentz algebra and the associated $S U(2) \otimes S U(2)$ symmetry of the Lorentz group [7].

The aim of our short theoretical paper is to contribute, in a new algebraic fashion, to a resolution of this important issue. For that purpose, we study again from scratch the spin and kinetic helicity of a fermion as described by the standard Dirac equation [6], which yet is appropriately generalized here. In particular, two novel aspects which we derive from the Dirac equation are:

1. Different representations of the quantization of the fermion spin, or choices of its basis, reveal three additional rotational so far hidden degrees of freedom.

\footnotetext{
a e-mail: marsch@ physik.uni-kiel.de (corresponding author)

be-mail: yasuhito.narita@oeaw.ac.at
} 
2. The notion of the kinetic helicity is generalized and can be connected to the rotation group $S O(3)$ in the real, and thus $S U(3)$ in the complex, three-dimensional space.

It turns out that three independent ways to express the kinetic helicity are found in connection with spin rotation in real space. The consequences of this result with respect to quantum field theory are found to be in compliance with the important Coleman-Mandula theorem [8]. Thus, mathematically speaking, the associated Lie group $S O(3)$ as adopted in our paper serves two physical applications, namely it first describes spin rotation, but then can naturally be extended and used for fermion colour (assigned to a triplet) mixing by $S U$ (3) as well, and thus may be identified with the empirical threefold colour degrees of freedom of the observed quarks. A close connection has been shown by Marsch and Narita $[9,10]$ to exist in the extended Dirac equation between the Clifford algebra of Lorentz invariance and the Lie algebra for the $S U(2)$ as well as the $S U(3)$ colour gauge group.

The outline of our paper is as follows: We start with the mass shell condition and revisit the fermion spin and its related kinetic helicity. First, a generalized helicity for the Dirac equation is considered. Then, various ways to express helicity are presented. Three independent variants are obtained in connection with rotation in real space. We assemble them into a generalized three-component fermion state vector, which encompasses three Dirac spinors corresponding to each of the three independent helicity degrees of freedom. We then identify this triple of state-vector components with the three quark colours.

\section{Mass shell condition, spin and helicity}

In this section, we revisit the Dirac equation [6], and in particular discuss the spin-related kinetic helicity. How does the fermion spin arise in that equation, and why do the Pauli matrices [11] occur in this context? Because their key property is that they permit to linearize the kinetic energy term. It goes with the momentum squared in the basic relativistic (and also non-relativistic) dispersion relation for a massive particle, which is given by

$$
E^{2}-\mathbf{p}^{2}=m^{2}=P^{\mu} P_{\mu},
$$

stating the mass shell condition for a free particle of mass $m$, energy $E$ and momentum p. We conveniently use units for which $c=1$ and $\hbar=1$. The relativistically covariant four-momentum operator is associated with the temporal or spatial derivative as:

$$
P_{\mu}=(E,-\mathbf{p})=\mathrm{i} \partial_{\mu}=\mathrm{i}\left(\partial_{t}, \partial_{\mathbf{x}}\right),
$$

according to quantum mechanics [4]. We abbreviate the contravariant space time coordinates $x^{\mu}=(t, \mathbf{x})$ by $x$.

Using the SU(2) generators of the three-dimensional rotation group, we can formally take the square root of the momentum term in Eq. (1). Namely, in terms of Pauli matrices, we obtain

$$
(\sigma \cdot \mathbf{p})^{2}=\mathbf{p}^{2} 1_{2}
$$

The symbol $1_{2}$ denotes the $2 \times 2$ unit matrix, and $\sigma_{\mathrm{x}}, \sigma_{\mathrm{y}}$, and $\sigma_{\mathrm{z}}$ the Pauli spin matrices:

$$
\sigma_{\mathrm{x}}=\left(\begin{array}{ll}
0 & 1 \\
1 & 0
\end{array}\right), \quad \sigma_{\mathrm{y}}=\left(\begin{array}{cc}
0 & -\mathrm{i} \\
\mathrm{i} & 0
\end{array}\right), \quad \sigma_{\mathrm{z}}=\left(\begin{array}{cc}
1 & 0 \\
0 & -1
\end{array}\right)
$$

satisfying the anti-commutative relation:

$$
\sigma_{i} \sigma_{j}+\sigma_{j} \sigma_{i}=2 \delta_{i j} 1_{2} .
$$


Obviously, this is how the fermion spin $S=\frac{1}{2} \sigma$, with the matrix three-vector $\sigma=$ $\left(\sigma_{\mathrm{x}}, \sigma_{\mathrm{y}}, \sigma_{\mathrm{z}}\right)$, emerges in quantum mechanics, namely via the spinor representation of the rotation group. We stress here that the Pauli matrices after Eq. (5) permit a matrix representation of the Euclidean metric of space, and thus to linearize Eq. (3). We shall dwell on this subject below.

Coming before as an interlude to Lorentz invariance, we remind the reader that historically speaking the prime task performed by Dirac [6] was to linearize the mass-shell condition Eq. (1) in order to obtain a Hamiltonian linear in the momentum. He solved this problem by introducing his famous $4 \times 4$ gamma matrices. Let us reconsider this problem, and for that purpose introduce the kinetic helicity operator as $H(\mathbf{p})=\sigma \cdot \mathbf{p}$. The mass shell condition can then in $4 \times 4$ matrix form be written as follows:

$$
\left(\sigma_{\mathrm{z}} \otimes 1_{2} E+\mathrm{i} \sigma_{\mathrm{y}} \otimes H(\mathbf{p})\right)^{2}=1_{4} m^{2},
$$

Therefore, we can define, either in the standard Dirac basis or in the chiral Weyl basis, the $4 \times 4$-matrix gamma four-vectors $(\otimes$ stands for the tensor product of the matrices involved) as follows:

$$
\gamma_{\mathrm{D}}^{\mu}=\left(\sigma_{\mathrm{z}} \otimes 1_{2}, \mathrm{i} \sigma_{\mathrm{y}} \otimes \sigma\right) \text {, or } \gamma_{\mathrm{W}}^{\mu}=\left(\sigma_{\mathrm{x}} \otimes 1_{2}, \mathrm{i} \sigma_{\mathrm{y}} \otimes \sigma\right) .
$$

In both versions, the same $\mathrm{i} \sigma_{\mathrm{y}}$ appears at the spin term. The Pauli matrices are all three connected via similarity transformations (see the Appendix). Therefore, by permutation of the Pauli matrices, one obtains four more possible versions of the gamma matrices as discussed in Marsch [5]. They are not in practical use; however, but in the SM the Weyl basis is the preferred choice. For both above forms of the gamma matrices, we restore the mass shell condition $\left(\gamma^{\mu} P_{\mu}\right)^{2}=m^{2} 1_{4}$, which allows one to linearize Eq. (1) in close analogy to Eq. (3), on which we concentrate now.

We followed here the historical path of Dirac [6], who started with the Lorentz-invariant scalar $m^{2}=P_{\mu} P^{\mu}$, which is the simplest Casimir operator of the Lorentz group. The other one is the Pauli-Lubanski operator $[14,15]$ not considered here. Of course, the modern derivation of the Dirac equation is at the outset exploiting the spinor representation of the Lorentz group $[4,7]$. We are not focusing here on the Lorentz group, but on the rotational invariance of the mathematical representation of the fermion spin, which as a vector has three degrees of freedom and can be written generally as $\boldsymbol{\sigma}(\boldsymbol{\theta})$, with the independent Euler angle vector $\boldsymbol{\theta}$ explained in detail in the subsequent Section 4. Using that quantity for the representation of $S U$ (2), one can of course then go the standard way of deriving the Lagrangian for a fermion by means of the spinor-field representation of the Lorentz group [4], as it can be constructed for any given $\gamma^{\mu}$.

So the "hidden symmetry" revealed here later lies in the free choice of the representation of the $S U$ (2) group, which involves the three Euler angles for the spin-axis orientation. The fermion spin is an independent physical degree of freedom and different from the fermion space-time four momentum (subjected to Lorentz invariance). This property becomes most obvious in the Wigner [7] state classification, where the Pauli-Lubanski operator is used and turns out to be equal to $-m^{2} s(s+1)$ (with the spin quantum number $s=1 / 2$ ) in the fermion rest frame.

\section{Helicity realizations linked by similarity transformations}

The main subsequent aim of our paper is to find out, what the possible physically different representations exist of the kinetic helicity operator, which we write down in standard form as: 


$$
H(\mathbf{p})=\sigma \cdot \mathbf{p}=\sigma_{\mathrm{x}} p_{\mathrm{x}}+\sigma_{\mathrm{y}} p_{\mathrm{y}}+\sigma_{\mathrm{z}} p_{\mathrm{z}} .
$$

Before we answer that question by the subsequent analysis, we like to mention here that we consider briefly the eigenfunctions of the helicity operator in Appendix. Manifestly, the mass shell condition Eq. (1) is invariant under the Lorentz transformation by definition. As the spin also does not enter in Eq. (1), the resulting energy dispersion relation of a free fermion, when taking the positive energy solution, is simply

$$
E(p)=\sqrt{m^{2}+p^{2}},
$$

and this energy of a free fermion is highly degenerate. For example, it is the same for all four degrees of freedom (particle, antiparticle, spin up, spin down) of the standard Dirac equation, or the eight fermions of the $S U(2) \otimes S U(4)$ symmetry octet, as described by the generalized Dirac equation derived by Marsch and Narita [12,13]. Since $H^{2}(\mathbf{p})=p^{2} 1_{2}$, it does not depend on the spin orientation either. Inspection of the basic definition in Eq. (8) may suggest that $H(\mathbf{p})$ is uniquely defined. Yet is this truly the case? The module of the squared fermion spin vector $\mathbf{S}^{2}$ equals $\frac{3}{4} 1_{2}$, and thus it is rotationally invariant. Three-dimensional Euclidean space is spanned by three independent orthogonal axes. Projected onto each of them, the three spin components may attain the values $\pm \frac{1}{2}$, yet only one of them at a time can according to quantum mechanics be sharply defined.

However, there exist these three independent options, which are lost in the information carried by kinetic helicity, which can only be either +1 for a right-handed or -1 for a lefthanded fermion, whatever its spin and momentum orientations may be in three-dimensional space. It has in fact eight octants with correspondingly eight $\left(2^{3}=8\right)$ possible sign pairs that are defined by the positive or negative signs associated with the three axes spanning three-dimensional space. For an illustration see Fig. 1. Consequently, helicity (where from now on $\mathbf{p}$ means the eigenvalue of the momentum operator) may be defined in various ways, for instance, as:

$$
\tilde{h}(\hat{\mathbf{p}})=\left( \pm \sigma_{\mathrm{x}}\right) \hat{\mathbf{p}}_{\mathrm{x}}+\left( \pm \sigma_{\mathrm{y}}\right) \hat{\mathbf{p}}_{\mathrm{y}}+\left( \pm \sigma_{\mathrm{z}}\right) \hat{\mathbf{p}}_{\mathrm{z}}
$$

whereby its square always yields $(\tilde{h}(\hat{\mathbf{p}}))^{2}=1_{2}$. Here, the momentum unit vector is defined as: $\hat{\mathbf{p}}=\mathbf{p} / p=(\sin \vartheta \cos \varphi, \sin \vartheta \sin \varphi, \cos \vartheta)$. For the eight sign combinations in Eq. (10)), the respective spin vector $\mathbf{S}$ points into the eight possible octants, which yet has no effect on the kinetic energy of the free fermion. So the sign at any sigma matrix is arbitrary and does not matter physically. But what about a cyclic permutation of the indices of the sigma matrices? Let us define the following (there are only these three) variants of $h(\hat{\mathbf{p}})$ :

$$
\begin{aligned}
& h_{\mathrm{xyz}}(\hat{\mathbf{p}})=\sigma_{\mathrm{x}} \hat{\mathbf{p}}_{\mathrm{x}}+\sigma_{\mathrm{y}} \hat{\mathbf{p}}_{\mathrm{y}}+\sigma_{\mathrm{z}} \hat{\mathbf{p}}_{\mathrm{z}}, \\
& h_{\mathrm{yzx}}(\hat{\mathbf{p}})=\sigma_{\mathrm{y}} \hat{\mathbf{p}}_{\mathrm{x}}+\sigma_{\mathrm{z}} \hat{\mathbf{p}}_{\mathrm{y}}+\sigma_{\mathrm{x}} \hat{\mathbf{p}}_{\mathrm{z}}, \\
& h_{\mathrm{zxy}}(\hat{\mathbf{p}})=\sigma_{\mathrm{z}} \hat{\mathbf{p}}_{\mathrm{x}}+\sigma_{\mathrm{x}} \hat{\mathbf{p}}_{\mathrm{y}}+\sigma_{\mathrm{y}} \hat{\mathbf{p}}_{\mathrm{z}} .
\end{aligned}
$$

So, the kinetic helicity is generalized from a scalar product type into a tensor-product type, allowing off-diagonal projection of the spin onto the momentum direction. The situation is illustrated in Fig. 1 that shows the three different choices of spin basis: one longitudinal type of spin orientation as described in Eq. (11) and two transverse types given in Eqs. (12) and Eq. (13).

By squaring these helicities, thereby exploiting the metric property in Eq. (3) of the Pauli matrices, it becomes obvious that the invariable result is $1_{2}$, as it should. The first is the original version of the normalized helicity, and the other two are related to it by two 
longitudinal

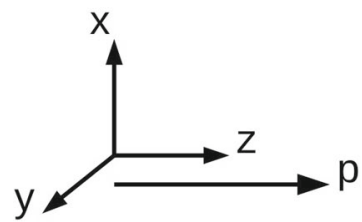

transverse 1

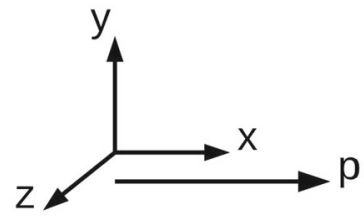

transverse 2

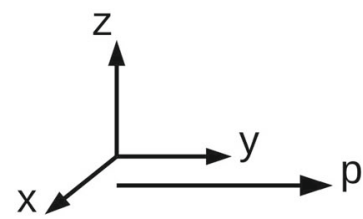

Fig. 1 Different basis choices for fermion spin with respect to the momentum direction

subsequent (out of three possible) unitary similarity transformations $X, Y$ and $Z$, which are defined and explained in Appendix.

Another triple of helicity operators is obtained by the subsequent singular application of the similarity transformations. Correspondingly, we define

$$
\begin{aligned}
& h_{\mathrm{x}}(\hat{\mathbf{p}})=X h(\hat{\mathbf{p}}) X^{-1}=+\sigma_{\mathrm{x}} \hat{\mathbf{p}}_{\mathrm{x}}+\sigma_{\mathrm{z}} \hat{\mathbf{p}}_{\mathrm{y}}-\sigma_{\mathrm{y}} \hat{\mathbf{p}}_{\mathrm{z}}, \\
& h_{\mathrm{y}}(\hat{\mathbf{p}})=Y h(\hat{\mathbf{p}}) Y^{-1}=-\sigma_{\mathrm{z}} \hat{\mathbf{p}}_{\mathrm{x}}+\sigma_{\mathrm{y}} \hat{\mathbf{p}}_{\mathrm{y}}+\sigma_{\mathrm{x}} \hat{\mathbf{p}}_{\mathrm{z}}, \\
& h_{\mathrm{z}}(\hat{\mathbf{p}})=Z h(\hat{\mathbf{p}}) Z^{-1}=+\sigma_{\mathrm{y}} \hat{\mathbf{p}}_{\mathrm{x}}-\sigma_{\mathrm{x}} \hat{\mathbf{p}}_{\mathrm{y}}+\sigma_{\mathrm{z}} \hat{\mathbf{p}}_{\mathrm{z}} .
\end{aligned}
$$

These expressions can also be written in terms of matrices, which better elucidates the nature of the similarity transformations involved as being equivalent to a rotation about one of the three orthogonal axes. These transformations are given explicitly in Appendix.

Finally, an important note of caution is in order here. The spin representation is twodimensional via the Pauli matrices, and thus, the two helicity eigenvalues of \pm 1 correspond to the positive and the mirrored negative orientation along any given of the three independent axes spanning real space. Therefore, only one set with three versions of the matrix helicity operator is to be accounted for. We are not obliged to make explicit use of the three algebraically different forms of the kinetic helicity, but may simply use the standard version $H(\mathbf{p})$ of Eq. (8).

However, there is a good physical reason to consider them all, because empirically we know that there are fermions, namely the famous quarks, which only differ by the three colour charges they are assumed to carry. This corresponds to the gauge symmetry group $S U$ (3), which leads to QCD. In a physically intuitive interpretation, the three colour degrees of freedom may simply reflect the three dimensions of real space. The resulting possible orientations of the fermion spin, which at a time can be defined sharply only with respect to one out of the three basic spatial axes.

As has been shown by Marsch and Narita [10] it is appropriate to define the subsequent extended Pauli matrix vector $\hat{\sigma}_{3}$ when considering the gauge group $S U(3)$. Thus, we may consider the, at a first sight apparently less trivial, version

$$
\hat{\boldsymbol{\sigma}}_{3}=\left(\left(\begin{array}{ccc}
\sigma_{\mathrm{x}} & 0 & 0 \\
0 & \sigma_{\mathrm{y}} & 0 \\
0 & 0 & \sigma_{\mathrm{z}}
\end{array}\right),\left(\begin{array}{ccc}
\sigma_{\mathrm{y}} & 0 & 0 \\
0 & \sigma_{\mathrm{z}} & 0 \\
0 & 0 & \sigma_{\mathrm{x}}
\end{array}\right),\left(\begin{array}{ccc}
\sigma_{\mathrm{z}} & 0 & 0 \\
0 & \sigma_{\mathrm{x}} & 0 \\
0 & 0 & \sigma_{\mathrm{y}}
\end{array}\right)\right) .
$$

It has the same algebraic properties as the Pauli matrix vector Eq. (3), but manifestly reflects the three degrees of freedom of $S U$ (3). In the top position of the matrix diagonal, we have already the three Pauli matrices. By adequately changing the basis for the middle and bottom positions, one can, however, transform their three components to attain the standard form as used in the top. Therefore, after adequate unitary transformations, one can obtain the equivalent but simpler definition $\hat{\sigma}_{3}=1_{3} \otimes \sigma$. It should be emphasized that such highly 
symmetric form as in Eq. (17) obtained by cyclic permutation is only possible for $S U$ (3), though, since the three dimensions of its matrices in fundamental representation correspond to the three axes spanning the real three-dimensional space, for which the three Pauli matrices provide the generators of the Pauli spinor representation of the rotation group. Finally, we may construct the helicity $3 \times 3$ matrix as:

$$
\hat{H}_{3}(\mathbf{p})=\hat{\boldsymbol{\sigma}}_{3} \cdot \mathbf{p} .
$$

This is unitarily equivalent to $1_{3} \otimes H(\mathbf{p})$, involving the original helicity of Eq. (8). The key result of this discussion then is that we obtain three colour degrees of freedom, which we can assemble into a triplet state vector. Marsch and Narita [10] studied the possible mathematical connections of the Clifford algebra with the $s u(N)$-Lie algebra, or in more physical terms the links between space-time symmetry (Lorentz invariance) and internal $S U(N)$ gaugesymmetry for a massive spin one-half fermion described by the Dirac equation. The related matrix algebra was worked out in particular for the gauge group $S U(3)$. They concluded that the extended Dirac equation based on the above generalized helicity $\hat{H}_{3}(\mathbf{p})$ is unitarily equivalent to the SM gauge theory for $S U(3)$ colour symmetry.

\section{Classical spin rotation matrix}

As shown in the previous section, we obtain three distinct helicity states for a generalized Dirac fermion. State mixing or change of the basis in Hilbert space is of course possible, since mixing is a most common phenomenon in quantum mechanics. But before we address this subject at the end of this section, it turns out that a physically intuitive and helpful description of what is going on can be obtained by considering first the rotation of the spin $\mathbf{S}=\left(S_{\mathrm{x}}, S_{\mathrm{y}}, S_{\mathrm{z}}\right)$ perceived as a classical vector in three-dimensional coordinate space. The generators of the related $S O(3)$ rotation group are the $3 \times 3$ matrices

$$
\tau_{\mathrm{x}}=\left(\begin{array}{ccc}
0 & 0 & 0 \\
0 & 0 & -\mathrm{i} \\
0 & \mathrm{i} & 0
\end{array}\right), \tau_{\mathrm{y}}=\left(\begin{array}{ccc}
0 & 0 & \mathrm{i} \\
0 & 0 & 0 \\
-\mathrm{i} & 0 & 0
\end{array}\right), \tau_{\mathrm{z}}=\left(\begin{array}{ccc}
0 & -\mathrm{i} & 0 \\
\mathrm{i} & 0 & 0 \\
0 & 0 & 0
\end{array}\right) .
$$

They are Hermitian and in cyclic order obey the general angular momentum algebra $\left[\tau_{\mathrm{x}}, \tau_{\mathrm{y}}\right]=$ $\mathrm{i} \tau_{\mathrm{z}}$. The phase factor associated with the $S O(3)$ group is $\exp (\mathrm{i} \boldsymbol{\tau} \cdot \boldsymbol{\theta})$, with a constant vector of three rotation angles assembled in $\boldsymbol{\theta}=\left(\theta_{\mathrm{x}}, \theta_{\mathrm{y}}, \theta_{\mathrm{z}}\right)$. Upon rotation the spin vector $\mathbf{S}$ changes, but its length or norm $\left(\mathbf{S}^{\dagger} \cdot \mathbf{S}\right)$ must remain invariant. The norm is not affected by a general phase, such when

$$
\mathbf{S} \rightarrow \mathbf{S}^{\prime}=\exp (\mathrm{i} \boldsymbol{\tau} \cdot \boldsymbol{\theta}) \mathbf{S}
$$

Written out explicitly, for example, the phase matrix for the x-component reads

$$
\exp \left(\mathrm{i} \tau_{\mathrm{x}} \theta_{\mathrm{x}}\right)=1_{3}+\left(\cos \left(\theta_{\mathrm{x}}\right)-1\right) \tau_{\mathrm{x}}^{2}+\mathrm{i} \sin \left(\theta_{\mathrm{x}}\right) \tau_{\mathrm{x}} .
$$

Similar expressions are obtained for the indices $x$ and y. Squaring the matrices in Eq. (19) yields

$$
\tau_{\mathrm{x}}^{2}=\left(\begin{array}{lll}
0 & 0 & 0 \\
0 & 1 & 0 \\
0 & 0 & 1
\end{array}\right), \tau_{\mathrm{y}}^{2}=\left(\begin{array}{lll}
1 & 0 & 0 \\
0 & 0 & 0 \\
0 & 0 & 1
\end{array}\right), \quad \tau_{\mathrm{z}}^{2}=\left(\begin{array}{lll}
1 & 0 & 0 \\
0 & 1 & 0 \\
0 & 0 & 0
\end{array}\right)
$$


Exploiting these matrices, we can cast the matrices for rotating a usual vector in threedimensional space about the $\mathrm{x}, \mathrm{y}$ and $\mathrm{z}$ axes in the explicit shapes

$$
\begin{aligned}
& R_{\mathrm{x}}\left(\theta_{\mathrm{x}}\right)=\exp \left(\mathrm{i} \tau_{\mathrm{x}} \theta_{\mathrm{x}}\right)=\left(\begin{array}{ccc}
1 & 0 & 0 \\
0 & c_{\mathrm{x}} & s_{\mathrm{x}} \\
0 & -s_{\mathrm{x}} & c_{\mathrm{x}}
\end{array}\right), \\
& R_{\mathrm{y}}\left(\theta_{\mathrm{y}}\right)=\exp \left(\mathrm{i} \tau_{\mathrm{y}} \theta_{\mathrm{y}}\right)=\left(\begin{array}{ccc}
c_{\mathrm{y}} & 0 & -s_{\mathrm{y}} \\
0 & 1 & 0 \\
s_{\mathrm{y}} & 0 & c_{\mathrm{y}}
\end{array}\right), \\
& R_{\mathrm{z}}\left(\theta_{\mathrm{z}}\right)=\exp \left(\mathrm{i} \tau_{\mathrm{z}} \theta_{\mathrm{z}}\right)=\left(\begin{array}{ccc}
c_{\mathrm{z}} & s_{\mathrm{z}} & 0 \\
-s_{\mathrm{z}} & c_{\mathrm{z}} & 0 \\
0 & 0 & 1
\end{array}\right) .
\end{aligned}
$$

As abbreviation we introduced $c_{\mathrm{x}}=\cos \left(\theta_{\mathrm{x}}\right)$, and $s_{\mathrm{X}}=\sin \left(\theta_{\mathrm{x}}\right)$, and similarly for the other indices $y$ and z. Note that the matrices in Eq. (A.12)of Appendix are obtained from Eq. (23), respectively, for clockwise rotation angles of ninety degrees. Apparently, the $R$ matrices are real and orthogonal, which means their transposed are their inverse matrices, $R^{T}=R^{-1}$, and they have a determinant of unity.

The three angles chosen above are just one possible set of the Euler angles. Most conveniently, we employ here the convention used in aviation. By three subsequent rotations, we can go from the original frame, in which the spin matrix vector in Eq. (4) was defined by its Cartesian components, into the spin's body frame of reference, where its arrow fixes the new positive $x$ axis. As the axes are thereby changing, we use the conventional names for the associated angles as follows: $\theta_{\mathrm{x}}$ is the yaw angle, $\theta_{\mathrm{y}}$ the pitch angle, and $\theta_{\mathrm{z}}$ the roll angle. The whole multiplied rotation matrix and its inverse then read:

$$
\begin{aligned}
R(\boldsymbol{\theta}) & =R_{\mathrm{x}}\left(\theta_{\mathrm{x}}\right) R_{\mathrm{y}}\left(\theta_{\mathrm{y}}\right) R_{\mathrm{z}}\left(\theta_{\mathrm{z}}\right), \\
R^{-1}(\boldsymbol{\theta}) & =R_{\mathrm{z}}^{-1}\left(\theta_{\mathrm{z}}\right) R_{\mathrm{y}}^{-1}\left(\theta_{\mathrm{y}}\right) R_{\mathrm{x}}^{-1}\left(\theta_{\mathrm{x}}\right),
\end{aligned}
$$

yielding $R(\boldsymbol{\theta}) R^{-1}(\boldsymbol{\theta})=1_{3}$. Now we can now rotate a vector, like, for example, the spin vector $\mathbf{S}=\frac{1}{2} \boldsymbol{\sigma}$, into any desired direction that is determined by $\boldsymbol{\theta}$ through transformation by means of the unitary operator $U(R)$, which is defined to act on the spin operator as follows: $\mathbf{S}(\boldsymbol{\theta})=U(R(\boldsymbol{\theta})) \mathbf{S} U^{-1}(R(\boldsymbol{\theta}))=R(\boldsymbol{\theta}) \mathbf{S}$, where on the right-hand side we have the normal matrix times vector multiplication. This transformation leaves the Lie algebra of the spin unchanged, i.e. we retain the commutator $\left[S_{\mathrm{x}}(\boldsymbol{\theta}), S_{\mathrm{y}}(\boldsymbol{\theta})\right]=\mathrm{i} S_{\mathrm{z}}(\boldsymbol{\theta})$. Consequently, also $\boldsymbol{\sigma}(\boldsymbol{\theta})$ obeys the metric condition in Eq. (5) for any angle $\boldsymbol{\theta}$.

As a result, we can use (in either the standard Dirac or chiral Weyl basis) the spin matrix $\boldsymbol{\sigma}(\boldsymbol{\theta})$ in the gamma-matrix four vectors defined in Eq. (7), i.e. we have

$$
\gamma_{\mathrm{D}}^{\mu}(\boldsymbol{\theta})=\left(\sigma_{\mathrm{z}} \otimes 1_{2}, \mathrm{i} \sigma_{\mathrm{y}} \otimes \sigma(\boldsymbol{\theta})\right)
$$

or similarly,

$$
\gamma_{\mathrm{W}}^{\mu}(\boldsymbol{\theta})=\left(\sigma_{\mathrm{x}} \otimes 1_{2}, \mathrm{i} \sigma_{\mathrm{y}} \otimes \boldsymbol{\sigma}(\boldsymbol{\theta})\right)
$$

In both expressions, the $\sigma_{\mathrm{y}}$ matrix appears at the spin term. For $\boldsymbol{\theta}=\mathbf{0}$, we retain the previous expressions of Eq. (7) for the gamma matrices. The kinetic helicity operator as defined via Eq. (A.1) may then generally be written as:

$$
h(\boldsymbol{\theta}, \hat{\mathbf{p}})=\boldsymbol{\sigma}(\boldsymbol{\theta}) \cdot \hat{\mathbf{p}}=\sigma_{\mathrm{x}}(\boldsymbol{\theta}) \hat{p}_{\mathrm{x}}+\sigma_{\mathrm{y}}(\boldsymbol{\theta}) \hat{p}_{\mathrm{y}}+\sigma_{\mathrm{z}}(\boldsymbol{\theta}) \hat{p}_{\mathrm{z}} .
$$


Thus, we seem to get an infinite set of helicities, depending on the continuous spin orientation angle. The six previous versions in Eqs. (11-13) and Eqs. (14-16) are retrieved from Eq. (27) for corresponding choices of the vector angle $\boldsymbol{\theta}$. However, according to the quantum mechanics of spin, it may only have one component being precisely defined at $\pm 1 / 2$ with respect to any given axis, in addition to its fixed module of $\mathbf{S}^{2}=3 / 4$. Therefore, we may only have three possible choices, in accord with three orthogonal axes spanning the real three-dimensional space, as we discussed extensively in the previous section. The axis configuration is fixed by the three Euler angles defining the "body frame" of the spin, corresponding to three independent degrees of freedom.

Consequently, let us return to the Hilbert space associated with these three colour degrees of freedom as described now by the triplet state vector $\Psi$, where $\Psi^{\dagger}=\left(\psi_{1}^{\dagger}, \psi_{2}^{\dagger}, \psi_{3}^{\dagger}\right)$, and whereby the index labels the colour kinds (synonymous to helicity variant) for each of the three Dirac spinors involved. Upon a basis change or rotation mediated by $R(\boldsymbol{\theta})$, the state vector will change, but its norm $\Psi^{\dagger} \Psi=\psi_{1}^{\dagger} \psi_{1}+\psi_{2}^{\dagger} \psi_{2}+\psi_{3}^{\dagger} \psi_{3}$ must remain invariant. It is also not affected by any phase change, like $\Psi \rightarrow \Psi^{\prime}=\exp (\mathrm{i} \tau \cdot \boldsymbol{\theta}) \Psi$.

\section{Conclusions}

This paper addresses the quark colour and the question of what might be at the origin of the well-established structure of the fermions, coming empirically as three types of coloured quarks and singular charged and neutral leptons. In a simple intuitive answer, one could say that the number of three colours simply reflects the three dimensions of coordinate space. As the fermion spin has the character of a vector but with matrix structure (i.e. with two internal degrees of freedom), its orientation in coordinate space is classically determined by the three Euler angles implying three degrees of freedom. Quantum mechanics permits only one spin component (in a given coordinate system) to be sharply determined at a time; however, the three intrinsic degrees of freedom remain. We propose in this paper that the fermion spin should generally be described by three different orientations of the basis, yielding three types of quantization: a longitudinal one with respect to the momentum direction and two transverse ones, as illustrated in Fig. 1.

They are subsumed in a generalized helicity triplet, which is connected with the $S O$ (3) rotation group, describing usually rotations in real vector space. The related symmetry group $S O(3)$ is suggested here to provide the means to change the colour basis, and thus to describe aside from spin rotation also colour mixing, which has the $S U(3)$ symmetry in the related complex three-dimensional Hilbert space, and thus is associated with the unitary transformation (rotation in Hilbert space) of the state vector consisting of the colour-related triplet of the Dirac spinors. This orientation knowledge is encoded in the generalized helicity, results in the triplet nature of the fermion, and thus may give rise to the colours of quarks.

Acknowledgements Eckart Marsch gratefully acknowledges the institutional support by the Extraterrestrial Physics Group headed by Robert Wimmer-Schweingruber at the Institute for Experimental and Applied Physics of Kiel University in Germany. He also appreciates the support by the Space Research Institute in Graz, Austria, where some ideas for this paper were developed.

Funding Open Access funding enabled and organized by Projekt DEAL.

Open Access This article is licensed under a Creative Commons Attribution 4.0 International License, which permits use, sharing, adaptation, distribution and reproduction in any medium or format, as long as you give appropriate credit to the original author(s) and the source, provide a link to the Creative Commons licence, and indicate if changes were made. The images or other third party material in this article are included in the 
article's Creative Commons licence, unless indicated otherwise in a credit line to the material. If material is not included in the article's Creative Commons licence and your intended use is not permitted by statutory regulation or exceeds the permitted use, you will need to obtain permission directly from the copyright holder. To view a copy of this licence, visit http://creativecommons.org/licenses/by/4.0/.

\section{Appendix: Similarity transformations of the helicity}

The eigenvalue equation of the kinetic helicity in Fourier space (where from now on $\mathbf{p}$ means the eigenvalue of the momentum operator) reads

$$
h(\hat{\mathbf{p}}) u_{ \pm}(\hat{\mathbf{p}})=(\sigma \cdot \hat{\mathbf{p}}) u_{ \pm}(\hat{\mathbf{p}})= \pm u_{ \pm}(\hat{\mathbf{p}}) .
$$

Both two-component Pauli eigenspinors [11] depend on the momentum unit vector, which is defined as: $\hat{\mathbf{p}}=\mathbf{p} / p=(\sin \vartheta \cos \varphi, \sin \vartheta \sin \varphi, \cos \vartheta)$. They have the form

$$
u_{+}(\hat{\mathbf{p}})=\left(\begin{array}{c}
\cos \frac{\vartheta}{2} \mathrm{e}^{-\frac{\mathrm{i}}{2} \varphi} \\
\sin \frac{\vartheta}{2} \mathrm{e}^{\frac{\mathrm{i}}{2} \varphi}
\end{array}\right), u_{-}(\hat{\mathbf{p}})=\left(\begin{array}{c}
-\sin \frac{\vartheta}{2} \mathrm{e}^{-\frac{\mathrm{i}}{2} \varphi} \\
\cos \frac{\vartheta}{2} \mathrm{e}^{\frac{\mathrm{i}}{2} \varphi}
\end{array}\right) .
$$

Here, the half angles of $\vartheta$ and $\varphi$ appear. The eigenvectors for the same $\hat{\mathbf{p}}$ are orthogonal to each other and normalized to a modulus of unity, and they obey the relation $u_{ \pm}(-\hat{\mathbf{p}})=\mathrm{i} u_{\mp}(\hat{\mathbf{p}})$. This is a consequence of the eigenvalue equation Eq. (A.1) which implies that $u_{ \pm}(\hat{\mathbf{p}})$, as an eigenvector of the helicity operator, corresponds to a right-handed, respectively, left-handed, screw with respect to the momentum direction. According to Eq. (A.2), we have the conditions of normalization $u_{ \pm}^{\dagger}(\hat{\mathbf{p}}) u_{ \pm}(\hat{\mathbf{p}})=1$ and orthogonality $u_{\mp}^{\dagger}(\hat{\mathbf{p}}) u_{ \pm}(\hat{\mathbf{p}})=0$. The dagger denotes as usually the transposed and complex conjugated vector or matrix.

We recall that it is convenient to introduce the spin-flip operator, $\tau=-\mathrm{i} \sigma_{\mathrm{y}} \mathbb{C}$, as defined in [12]. When operating with it on the above eigenspinors, this leads to

$$
\tau u_{ \pm}(\hat{\mathbf{p}})= \pm u_{\mp}(\hat{\mathbf{p}})=\mp \mathrm{i} u_{ \pm}(-\hat{\mathbf{p}}),
$$

i.e. it connects the eigenfunctions of opposite helicity. We recall that $\mathbb{C}$ denotes the complex conjugation operator, and that $\tau$ is anti-Hermitian and obeys $\tau^{2}=-1_{2}$. It defines the charge conjugation operator $\mathcal{C}=\gamma \tau$ for the Dirac equation.

There exist three obvious similarity transformations for $h(\hat{\mathbf{p}})$, which operate on the sigma matrices as follows:

$$
\begin{aligned}
X \sigma_{\mathrm{y}} X^{-1}=+\sigma_{\mathrm{z}} ; & X \sigma_{\mathrm{z}} X^{-1}=-\sigma_{\mathrm{y}}, \\
Y \sigma_{\mathrm{x}} Y^{-1}=-\sigma_{\mathrm{z}} ; & Y \sigma_{\mathrm{z}} Y^{-1}=+\sigma_{\mathrm{x}}, \\
Z \sigma_{\mathrm{x}} Z^{-1}=+\sigma_{\mathrm{y}} ; & Z \sigma_{\mathrm{y}} Z^{-1}=-\sigma_{\mathrm{x}} .
\end{aligned}
$$

The corresponding definitions are

$$
\begin{array}{ll}
X^{ \pm 1}=\frac{1}{\sqrt{2}}\left(1 \mp \mathrm{i} \sigma_{\mathrm{x}}\right), & X^{+1} X^{-1}=1_{2} \\
Y^{ \pm 1}=\frac{1}{\sqrt{2}}\left(1 \mp \mathrm{i} \sigma_{\mathrm{y}}\right), & Y^{+1} Y^{-1}=1_{2} \\
Z^{ \pm 1}=\frac{1}{\sqrt{2}}\left(1 \mp \mathrm{i} \sigma_{\mathrm{z}}\right), & Z^{+1} Z^{-1}=1_{2}
\end{array}
$$

All similarity transformations are unitary, i.e. for example, $\left(X^{ \pm 1}\right)^{\dagger}=X^{\mp 1}$. The two new variants Eq. (12) and Eq. (13), stemming originally from a cyclic permutation of the Pauli 
matrices appearing in $h(\hat{\mathbf{p}})$, are thus obtained by application of the combined similarity transformations:

$$
\begin{aligned}
h_{\mathrm{yzx}}(\hat{\mathbf{p}}) & =Z\left(X h(\hat{\mathbf{p}}) X^{-1}\right) Z^{-1} \\
& =X\left(Y h(\hat{\mathbf{p}}) Y^{-1}\right) X^{-1} \\
& =Y\left(Z h(\hat{\mathbf{p}}) Z^{-1}\right) Y^{-1} \\
h_{\mathrm{zxy}}(\hat{\mathbf{p}}) & =Y^{-1}\left(X^{-1} h(\hat{\mathbf{p}}) X\right) Y \\
& =Z^{-1}\left(Y^{-1} h(\hat{\mathbf{p}}) Y\right) Z \\
& =X^{-1}\left(Z^{-1} h(\hat{\mathbf{p}}) Z\right) X .
\end{aligned}
$$

The eigenspinors associated with $h_{\mathrm{yzx}}$ and $h_{\mathrm{zxy}}$ are obtained by operation of the transformation $X Y$, respectively, $Z^{-1} Y^{-1}$ on the $u_{ \pm}(\hat{\mathbf{p}})$ as given in Eq. (A.2).

These expressions Eqs. (14-16) can also be written in terms of matrices, which better elucidates the nature of the similarity transformation as being equivalent to a rotation about one of the three orthogonal axes. Thus, we may write $h(\hat{\mathbf{p}})=\boldsymbol{\sigma} \cdot 1_{3} \cdot \hat{\mathbf{p}}$, and similarly $h_{\mathrm{x}, \mathrm{y}, \mathrm{z}}(\hat{\mathbf{p}})=\sigma \cdot M_{\mathrm{x}, \mathrm{y}, \mathrm{z}} \cdot \hat{\mathbf{p}}$. The matrices appearing here are just the real $3 \times 3$ matrices (with unit determinant) for a rotation by ninety degrees about the $x, y$ and $z$ axis, respectively, and can be written as:

$$
M_{\mathrm{x}}=\left(\begin{array}{ccc}
1 & 0 & 0 \\
0 & 0 & -1 \\
0 & 1 & 0
\end{array}\right), M_{\mathrm{y}}=\left(\begin{array}{ccc}
0 & 0 & 1 \\
0 & 1 & 0 \\
-1 & 0 & 0
\end{array}\right), M_{\mathrm{z}}=\left(\begin{array}{ccc}
0 & -1 & 0 \\
1 & 0 & 0 \\
0 & 0 & 1
\end{array}\right) .
$$

Like in the previous case, we get the eigenspinors associated with $h_{\mathrm{x}, \mathrm{y}, \mathrm{z}}$ by operation of the transformation $X, Y, Z$, respectively, on the eigenspinor $u_{ \pm}(\hat{\mathbf{p}})$ as given in Eq. (A.2). Apparently, we have in this way obtained two equivalent sets (with three versions for each) of the kinetic helicity operator in Eq. (8). As a similarity transformation means rotation about one of the six Euclidean axes, both helicity sets are again connected in this way, yielding $Z^{-1} h_{\mathrm{yzx}}(\hat{\mathbf{p}}) Z=h_{\mathrm{x}}(\hat{\mathbf{p}}), X^{-1} h_{\mathrm{yzx}}(\hat{\mathbf{p}}) X=h_{\mathrm{y}}(\hat{\mathbf{p}})$, and $Y^{-1} h_{\mathrm{yzx}}(\hat{\mathbf{p}}) Y=h_{\mathrm{z}}(\hat{\mathbf{p}})$. Consequently, when we write the helicities after Eqs. (11-13) as $h_{\mathrm{xyz}}(\hat{\mathbf{p}})=\sigma \cdot M_{\mathrm{xyz}} \cdot \hat{\mathbf{p}}$, and likewise in cyclic permutation, we find that $M_{\mathrm{xyz}}=1_{3}, M_{\mathrm{yzx}}=M_{\mathrm{z}} M_{\mathrm{x}}$, and $M_{\mathrm{zxy}}=M_{\mathrm{y}}^{-1} M_{\mathrm{x}}^{-1}$. All $M$-matrices have a determinant of unity and thus correspond to rotations.

\section{References}

1. H. Machner, Einführung in Die Kern- Und Elementarteilchenphysik (Wiley-VCH Verlag, Weinheim, Germany, 2005)

2. M. Kaku, Quantum Field Theory (Oxford University Press, New York, USA, A Modern Introduction, 1993)

3. M.E. Peskin, D.V. Schroeder, An Introduction to Quantum Field Theory (Addison-Wesley Publishing Company, Reading, Massachusetts, USA, 1995)

4. M.D. Schwartz, Quantum Field Theory and the Standard Model (Cambridge University Press, Cambridge, UK, 2014)

5. E. Marsch, Fermion colour and flavour originating from multiple representations of the Lorentz group and Clifford algebra. Phys. Sci. Int. J. 23(3), 1-13 (2019)

6. P.A.M. Dirac (1928) The quantum theory of the electron. Proc. Roy. Soc. Lond. Ser. A Math. Phys. Sci. 117, 610 .

7. E. Wigner, On unitary representations of the inhomogeneous Lorentz group. The Ann. Math. 40(1), 149 (1939). https://doi.org/10.2307/1968551

8. S. Coleman, J. Mandula, All possible symmetries of the S matrix. Phys. Rev. D 159, 1251 (1967)

9. E. Marsch, Y. Narita, Dirac equation based on the vector representation of the Lorentz group. Eur. Phys. J. Plus 135, 782 (2020). https://doi.org/10.1140/epjp/s13360-020-00798-7 
10. E. Marsch, Y. Narita, Connecting in the Dirac equation the Clifford algebra of Lorentz invariance with the Lie algebra of $S U(N)$ gauge symmetry. Symmetry 1, 475 (2021). https://doi.org/10.3390/sym13030475

11. W. Pauli, Zur Quantenmechanik des magnetischen Elektrons. Z. Physik 43, 601 (1927). https://doi.org/ 10.1007/BF01397326

12. E. Marsch, Y. Narita, Fermion unification model based on the intrinsic SU(8) symmetry of a generalized Dirac equation. Front. Phys. 3, 82 (2015). https://doi.org/10.3389/fphy.2015.00082

13. E. Marsch, Y. Narita, Fundamental fermion interactions via vector bosons of unified $S U(2) \otimes S U(4)$ gauge fields. Front. Phys. 4, 5 (2016). https://doi.org/10.3389/fphy.2016.00005

14. J.K. Lubanśki, Sur la theorie des particules élémentaires de spin quelconque. I, Phys. 9, 310-324 (1942). https://doi.org/10.1016/S0031-8914(42)90113-7

15. J.K. Lubanśki, Sur la theorie des particules élémentaires de spin quelconque. II, Phys. 9, 325-338 (1942). https://doi.org/10.1016/S0031-8914(42)90114-9 